\title{
Developing new hepatitis B immunisation strategies
}

\author{
A J Zuckerman
}

\begin{abstract}
The four main approaches to immunisation against hepatitis $B$ are: vaccination of high risk babies; universal adolescent immunisation; universal infant immunisation; and vaccination of everybody. Universal antenatal screening would permit identification of carrier mothers and immunisation of their babies. Vaccination of adolescents would provide protection close to the time when risk of exposure increases, and could be delivered as part of a wider package on health education. Universal vaccination of infants is the best option because it is known that vaccines can be delivered to babies and it is more acceptable to parents. Development of a combined hepatitis B-diphtheria-pertussis-tetanus vaccine would be beneficial. The identification of hepatitis $B$ antibody escape mutants is of concern because of the implications for vaccine efficacy. Altered or absent expression of the hepatitis B virus (HBV) antigenic group determinant $a$ may allow infection even in subjects who have responded previously to vaccination.

(Gut 1996; 38 (suppl 2): S60-S62)
\end{abstract}

Keywords: hepatitis B, immunisation strategies, hepatitis B antibody escape mutants.

Although hepatitis B is a public health problem throughout the world and is preventable, a consensus on immunisation strategies is notable by its absence. Current policies in many countries have had little influence on the epidemiology of this important infection. The strategy of selective immunisation of groups at 'high risk' of infection has had little impact on hepatitis B outside, for example, health care personnel. The World Health Organisation has set a target of global control of hepatitis $B$, and recommended that all countries integrate hepatitis $B$ vaccine into their national immunisation programmes by 1997 . Some 75 countries, including Italy, France, Canada, and the USA have introduced such programmes to date.

Royal Free Hospital School of Medicine, London

A J Zuckerman

Correspondence to: Professor A J Zuckerman, Director, World Health Organisation Collaboratin Centre for Reference and Research on Viral Diseases Dean, Royal Free Hospital School of Medicine, London NW3 2 PF. Approaches to immunisation

The four main approaches to immunisation are as follows ${ }^{1}$ :

- Vaccination of 'high risk' babies, as defined below;

- Universal adolescent immunisation;

- Universal infant immunisation;

- Vaccination of everybody.
VACCINATION OF HIGH RISK BABIES

There is strong support for the introduction of universal antenatal screening to identify hepatitis B carrier mothers and permit immunisation of their babies. It is important that any other strategies do not interfere with the delivery of vaccine to this group, whose immunisation will have the greatest impact in reducing the number of new hepatitis $B$ carriers. For children outside this group, it is difficult to estimate the lifetime risk of acquiring a hepatitis infection, and the remaining three approaches listed should be considered. ${ }^{1}$

\section{VACCINATION OF ADOLESCENTS}

Vaccination of adolescents would provide protection against hepatitis $B$ at a time close to the time when 'risk behaviour' would expose adolescents to infection. Vaccination at this age could be delivered as part of a wider package on health education in general, including sex education, risk of AIDS, dangers of drug abuse and smoking, and the benefits of a healthy diet and life style.

There are several problems with this approach, including the following:

- Persuading parents to accept vaccination of their children against an infection that is also transmitted by the sexual route may not be easy, and may well be a problem they do not wish to consider at that time;

- Ensuring that a full course of three doses is given may be difficult;

- There would be difficulty evaluating and monitoring vaccine cover. The systems for monitoring uptake of vaccine in this age group may not operate efficiently.

\section{VACCINATION OF INFANTS}

The advantages of universal infant vaccination are as follows:

- It is known that vaccination can be delivered to babies;

- Parents are likely to accept vaccination against hepatitis $\mathbf{B}$ along with other childhood vaccinations without reference to sexual behaviour.

The disadvantages of this approach include the following:

- It is uncertain whether immunity would remain until exposure occurred in later life. It is possible that this would become less of a problem as more people were vaccinated, thereby reducing the chance of exposure to infection;

- The introduction of an additional childhood vaccination may reduce the uptake of other 
childhood vaccinations. This problem would be avoided if hepatitis $B$ vaccine could be delivered in a combined vaccine together with diphtheria-pertussis-tetanus immunogens. Such preparations are under evaluation.

\section{RECOMMENDATION}

Vaccination of infants is preferable to vaccination of adolescents as there are sufficient mechanisms to ensure, monitor, and evaluate cover. A booster dose could be given in early adolescence, combined with a health education package. A rolling programme could be introduced, giving priority to urban areas.

\section{Hepatitis $B$ antibody escape mutants} The identification of hepatitis $B$ antibody escape mutants may have important implications for vaccine efficacy and, in due course, for the safety of blood transfusions and transplants. Of particular importance are viruses with altered or absent expression of the group antigenic determinant $a$.

\section{EPITOPE $a$}

Production of antibodies to epitope $a$ mediates cross protection against all hepatitis $B$ virus (HBV) subtypes, as has been shown by challenge with a second subtype of the virus after recovery from an initial experimental infection. The epitope $a$ is located in the region of amino acids $124-148$ of the major surface protein, and seems to have a double loop conformation. A monoclonal antibody that recognises a region within this $a$ epitope is capable of neutralising the infectivity of $\mathrm{HBV}$, and competitive inhibition assays using the same monoclonal antibody show that equivalent antibodies are present in the sera of subjects immunised with either plasma derived or recombinant hepatitis $\mathbf{B}$ vaccine.

During a study of the immunogenicity and efficacy of hepatitis B vaccines in Italy, a number of subjects who had apparently mounted a successful immune response to the surface antigen of the virus (that is, became anti-HBs positive) later became infected with HBV. These cases were characterised by the coexistence of non-complexed anti-HBs and hepatitis B surface antigen ( $\mathrm{HBsAg}$ ) and, in 32 of 44 vaccinated subjects, there were other markers of HBV replication. ${ }^{2}$ Furthermore, analysis of the antigen using monoclonal antibodies suggested that the $a$ epitope was either absent or masked by antibody. Subsequent sequence analysis of the virus from one of these cases showed a mutation in the nucleotide sequence encoding the $a$ epitope, the consequence of which was a substitution of arginine for glycine at amino acid position $145 .^{3}$

The region in which this mutation occurs is an important virus epitope to which vaccine induced neutralising antibody binds, as discussed above. The mutant virus is not neutralised by antibody to this specificity. It can replicate as a competent virus, implying that the amino acid substitution does not change the attachment of the virus to the liver cell. This has been confirmed in experimental transmission studies at the National Institutes of Health in the USA. ${ }^{4}$

\section{GEOGRAPHICAL DISTRIBUTION}

There is now considerable evidence for a wide geographical distribution of the point mutation in HBV from guanosine to adenosine at position 587, resulting in an amino acid substitution at position 145 from glycine to arginine in the highly antigenic group determinant $a$ of the surface antigen. This stable mutation has been found in viral isolates from children several years later and it has been described in Italy, Singapore, Japan, and Brunei. ${ }^{5}$ It has also been found in liver transplant recipients with hepatitis B in the United States, Germany, and the United Kingdom who had been treated with specific hepatitis B immunoglobulin or humanised hepatitis B monoclonal antibody. ${ }^{5}$

During a study in progress in Singapore, three groups of babies were immunised against hepatitis B: 50 babies born to mothers without $\mathrm{HBsAg}$ and 600 born to mothers with $\mathrm{HBsAg}$ but without hepatitis $\mathrm{B}$ e antigen ( $\mathrm{HBeAg}$ ) were immunised successfully. However, among the 600 babies born to mothers with HBsAg and $\mathrm{HBeAg}$, there were 40 vaccine failures, and all were positive for $\mathrm{HBsAg}$ and core antibody. Direct sequencing has been completed for 26 isolates from these 40 infants. Fifteen had wild type sequences, and serological profiles usually indicated in utero infection. However, the other 11 had variant sequences namely, the 145 glycine to arginine variant alone (four cases) or with alanine at position 144 (in one twin), or other changes yet to be evaluated. ${ }^{6}$

Variants of HBV with altered antigenicity of the envelope protein show that this virus is not as antigenically singular as previously believed and that humoral escape mutation can occur in vivo. There are two important causes for concern: firstly, failure to detect $\mathrm{HBsAg}$ may lead to HBV transmission through donated blood or organs; secondly, the mutated HBV may infect subjects who are anti-HBs positive after immunisation. Mutants, variants, altered genotypes, and unusual strains are now being sought in many laboratories. The emergence of escape mutants may necessitate the modification of currently available hepatitis $B$ vaccines and the design of new hepatitis $B$ vaccines.

1 Proposal drafted by JE Banatvala, E Boxall, J Heptonstall, AJ Zuckerman, London: December 1991.

2 Zanetti AR, Tanzi E, Manzillo G, et al. Hepatitis B variant in Europe. Lancet 1988; ii: 1132-3.

3 Carman WF, Zanetti AR, Karayiannis P, et al. Vaccine induced escape mutant of hepatitis B virus. Lancet 1990; 336: 325-9.

4 Ogata N, Miller RG, Ishak KG, Zanetti AR, Purcell RH. Genetic and biological characterization of two hepatitis $B$ virus variants: a precore mutant implicated in fulminant virus variants: a precore mutant implicated in fulminant hepatitis and a surface mutant resistant to immunoprophylaxis. In. Nishioka K, Suzuki $\mathrm{H}$, Mishiro S, Osa T, eds. Viral hepatitis and liver disease. Tokyo: Springer-Verlag,

5 Zuckerman AJ, Harrison TJ, Oon C-J. Mutations in the S region of hepatitis B virus. Lancet 1994; 343: 737-8.

6 Oon C-J, Lim G-K, Ye Z, et al. Molecular epidemiology of hepatitis B virus variants in Singapore. Vaccine 1995; 13: 699-702. 


\section{Discussion}

Gust: Are there any data on what happens to non-human primates that have been immunised with the existing vaccine when they are challenged with the escape mutant?

Zuckerman: There was a study carried out at the NIH, but it did not mimic the situation that exists in real life. In this study, primates which had been vaccinated previously were challenged with the mutant virus and did not develop infection. Ideally, the mutant virus should be given at the same time as either $\mathrm{HBIg}$ or the vaccine.

Gust: From a public health point of view, the original NIH experiment is still an important study, because it suggests that people who are already immunised will also be protected against the mutant virus.

Zuckerman: It is reassuring to a point, although further studies need to be done.

Goudeau: Is a vaccine being developed to include the 145 mutant?

Zuckerman: A vaccine has been prepared and is available but has not yet been distributed because we do not yet know the extent of this problem. There are other mutations although they do not seem to be as important or as common as the 145 mutation. The 141 mutation in Gambia also seems to be driven by the immunisation programme and apparently involves about $10 \%$ of the children, but further follow up is needed.

Torres: In people who have previously been vaccinated with the original hepatitis $B$ vaccine, would the immunological response to the new vaccine be any different to that in previously unvaccinated people?

Zuckerman: Data are not available thus far. 